University for Business and Technology in Kosovo

UBT Knowledge Center

UBT International Conference

2013 UBT International Conference

Nov 1st, 5:30 PM - 5:45 PM

\title{
Comparison of local image descriptors for plant identification from leaf images
}

Lameski Petre

University of Sts Cyril and Methodius, petre.lameski@finki.ukim.mk

Zdravevski Eftim

University of Sts Cyril and Methodius, eftim.zdravevski@finki.ukim.mk

Kulakov Andrea

University of Sts Cyril and Methodius, andrea.kulakov@finki.ukim.mk

Follow this and additional works at: https://knowledgecenter.ubt-uni.net/conference

Part of the Computer Sciences Commons, and the Engineering Commons

\section{Recommended Citation}

Petre, Lameski; Eftim, Zdravevski; and Andrea, Kulakov, "Comparison of local image descriptors for plant identification from leaf images" (2013). UBT International Conference. 71.

https://knowledgecenter.ubt-uni.net/conference/2013/all-events/71

This Event is brought to you for free and open access by the Publication and Journals at UBT Knowledge Center. It has been accepted for inclusion in UBT International Conference by an authorized administrator of UBT Knowledge Center. For more information, please contact knowledge.center@ubt-uni.net. 


\title{
Comparison of local image descriptors for plant identification from leaf images
}

\author{
Lameski Petre, Zdravevski Eftim, Kulakov Andrea \\ University of Sts Cyril and Methodius - Skopje,Macedonia \\ Faculty of Computer Science and Engineering \\ \{petre.lameski, eftim.zdravevski, andrea.kulakov\}@finki.ukim.mk
}

\begin{abstract}
Abs tract. In this paper we present several descriptors used for the task of Plant recognition based on the images of leaves. The set of descriptors include texture based descriptors, fractal descriptors as well as some of the state of the art descriptors for image retrieval and object recognition in images. The descriptors are generated from the leaf images taken from single leaves on homogenous background. The descriptors are then used for training classifiers from a dataset of leaf images. The comparis on of the obtained results will be presented in this paper.
\end{abstract}

Key words: recognition, images, fractal, pixel

\section{Introduction}

The problem of plant identification and classification using images is a well-known problem in the scientific community and there are a lot of examples of usage of image processing techniques for solving this problem. There are many ongoing researches regarding plant identification from leaf images, plant images. Many descriptors that are also used in other image classification tasks are also used for the plant classification and identification task. The descriptors used to describe the image content and then classify the images by their content can be divided in several types based on the methods used for their extraction. The descriptors can be local or global descriptors. Local descriptors are generated by processing local image points or patches and global descriptors that are usually a histogram of local descriptors are used for describing the whole image. Based on the method of extraction, image descriptors can be divided in several groups: Texture based descriptors, Edge Histogram descriptors, Shape descriptors, Fractal Descriptor, etc.

Texture based descriptors use the image texture characteristics on local areas of the image. The image texture is represented by the pixel intensities, and these descriptors are generated by processing the pixel intensities and creating some transformation from those values in a local area in the image. One such descriptor is the Local Binary Pattern descriptor [1]. The Local Binary Pattern descriptor is generated by comparing pixels intensity of an intensity image with its neighboring pixels. For the purposes of our experiments we use a histogram of such local descriptors for the whole image of the leaf. Shape Context Descriptors take into account the shapes of the objects. These descriptors are usually used for object detection based on a transformation of their shape characteristics. One such descriptor is described in [2]. This descriptor uses image for which edges are first detected. It counts the point's distribution in predefined areas of the image and uses this information to generate a descriptor of the image.

Edge histogramdescriptors are used to describe the object characteristics in localpatches of the image. One such descriptor is the HOG (Histogram of oriented gradients) [3]. This descriptor is first introduced for pedestrian detection in images. Since we are working with images of single leaves, we will generate this descriptor for the fill area of the leaf, or in our case for the full image.

Fractal descriptors use the Fractal dimension of the image intensities to describe the image content. One such descriptor that is extracted by using extracted by using Segmentation-based FractalTexture Analys is is described in [4].

We use these descriptors to extract the information from the leaf images and then use them to train a Multilayer perceptron classifier. The descriptors used for this task are generated from the full content of each image of the leaves. The experimental setup is described in chapter 2. Chapter 3 gives the obtained results fromthe experiment and chapter 4 gives a short conclusion based on the obtained result. 


\section{Experimental Setup}

For this experiment, we used a dataset of single leaf images from various plants. Information on the dataset and the dataset files can be downloaded from [5]. The dataset is consisted of single leafimages on a homogenous background from 220 different plants. The total number of images in the dataset is 16683 images. Each plant is represented by 20 to over one hundred of images. We didn't preprocess the images of the dataset to normalize the amount of images per plant type. For each image, we first extracted each of the described descriptors. The Histogram of oriented gradients (HOG) descriptor was extracted for the whole image. Each descriptor of the images is represented by a vector of 81 values that contain the histogram count. The histogram was taken by dividing the image in 3 by 3 overlapping windows and the combined histogram of each of the windows gave the total descriptor for the image containing 81 values. For this experiment we used the implementation of HOG from [7].

The Fractal descriptor was extracted using Segmentation-based Fractal Texture Analysis algorithm (SFTA). We used the same implementation used for the work described in [4]. The SFTA descriptor used in our work is represented by 12 values vector for each image.

For extraction of the Local Binary Pattern descriptor (LBP) used in this work we used implementation of CLBP that generates a histogram of Local Binary Patterns for the full image. This implementation can be found in [8]. Each feature vector representing an image that was generated by the CLBP algorithm contains 118 values.

The Shape Context Descriptor was implemented based on [2]. We used our own implementation of this descriptor. The implementation first finds the edges of the image using the Canny edge detection algorithm [9]. From the edge image, a binary image is formed. We count the pixels that represent edge in each area around the center of the images. The areas are shown in Figure 1:

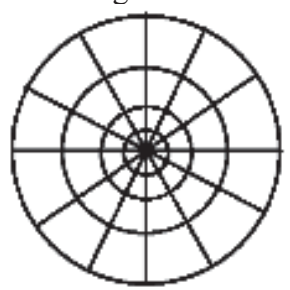

Fig. 1 Areas for point counting in the Shape Context Descriptor extraction

The total number of counts that generates the Shape Context Descriptor is 48 and that gives the dimension of the feature extracted from the image.

We used WEKA [6] data mining tool to train a Multilayer perceptron classifier for each descriptor type. The classifier was trained using $66 \%$ of the data and $33 \%$ were used for evaluation. The classifier implementation was provided from the WEKA data mining tool. The same parameters for the Multilayer perceptron classifier were used for each of the four descriptors. Each run was repeated three times and the average precisions were taken for result.

\section{Results}

After the models were created and the tests were run, we obtained the average precision value fromthe classification for each feature descriptor type. For the evaluation we used 3 fold cross validation. The dataset was divided in 3 separate groups of features. 2 groups were used for training the model and one group for testing the model performance. The obtained results are shown in Table 1.

Table 1. Average precision for each image descriptor

\begin{tabular}{lllll}
\hline Descriptor & HOG & SFTA & CLBP & Shape Context \\
Avg. Precision & $61.6556 \%$ & $49.8471 \%$ & $75.4301 \%$ & $20.1103 \%$ \\
\hline
\end{tabular}


The best performance in view of precision was shown by the Local Binary Pattern descriptor. It significantly outperforms the other descriptor types especially the Shape Context descriptor.

\section{Conclusion}

From the obtained results on this dataset, it can be seen that the texture descriptor gives the best result for leafimages classification and thus plant recognition based on the images of their leaves. This result shows that the analysis of the leaves texture gives a good direction towards generating a good feature descriptor for leaves classification for the task of plant identification. The Histogram of oriented gradients and the Fractal descriptor also show promising results. The Fractal descriptor also had the lowest vector dimension of only 12 values to describe the image. The Shape Context descriptor showed the worst performance. This lack of performance might be caused by the shape context descriptor very bad performance on small rotation. The shape context descriptor is also focused on the form of the leaves. Since some of the leaves are very similar in form, the shape context would be unable to make a descriptor for those classes of leaves that can be separable enough for the classifier to be able to make a good classification model. Based on the results it is possible to combine multiple descriptors and to build the model based on a more complex descriptor of the images that would contain different descriptors. We plan to experiment with this in future work. Also the currently generated descriptors might be used with different classifiers to see which classifier would be able to generate the best model for plant identification based on the leaf images. The main goal of this work was to find a good descriptor for image classification that can be used of plant recognition based on the image of a single leaf from the plant. This would allow us to build an autonomous robotic system that is able to segment the image and extract an image from a single leaf of some plant of interest and then recognize that plant based on the leaf images model that is previously trained.

\section{Acknowledgements}

This work was partially financed by the Faculty of Computer Science and Engineering at the "Ss. Cyril and Methodius University in Skopje", Skopje, R. Macedonia.

\section{References}

1. Z. Guo , L. Zhang and D. Zhang "A completed modeling of local binary pattern operator for texture classification", IEEE Trans. Image Process., vol. 19, no. 6, pp.1657 -1663 2010

2. S. Belongie, J. Malik, and J. Puzicha (April 2002). "Shape Matching and Object

3. Recognition Using Shape Contexts". IEEE Transactions on Pattern Analys is and Machine Intelligence 24 (24): 509-521. doi:10.1109/34.993558

4. Dalal, N.; Triggs, B., "Histograms of oriented gradients for human detection," Computer Vision and Pattern Recognition, 2005. CVPR 2005. IEEE Computer Society Conference on , vol.1, no., pp.886,893 vol. 1, 25-25 June 2005 doi: 10.1109/CVPR.2005.177

5. Costa, A. F., G. E. Humpire-Mamani, A. J. M. Traina. 2012. "An Efficient Algorithm for Fractal Analysis of Textures." In SIBGRAPI 2012 (XXV Conference on Graphics, Patterns and Images), 39-46, Ouro Preto, Brazil.

6. http://www.intelengine.cn/English/dataset/ (Last accessed 15.10.2013)

7. http://www.cs.waikato.ac.nz/ml/weka/ (Last accessed 15.10.2013)

8. O. Ludwig, D. Delgado, V. Goncalves, and U. Nunes, "Trainable Classifier-Fusion Schemes: An Application To Pedestrian Detection," In: 12th International IEEE Conference On Intelligent Transportation Systems, 2009, St. Louis, 2009. V. 1. P. 432-437. http://www.cse.oulu.fi/CMV/Downloads/LBPMatlab (Last accessed 15.10.2013)

9. Canny, J., A Computational Approach To Edge Detection, IEEE Trans. Pattern Analysis and Machine Intelligence, 8(6):679-698, 1986. 